\section{Numb chin syndrome: an ominous clinical sign}

\author{
F. Ryba, ${ }^{1}$ S. Rice ${ }^{2}$ and I. L. Hutchison ${ }^{3}$
}

IN BRIEF
- Highlights a rare but important clinical
finding associated with significant
morbidity and mortality.
- Presentation to the general dental
practitioner is common and may be
under-investigated.
- Provides an overview of the most
appropriate immediate investigations.

Numb chin syndrome (NCS) is a rare but important clinical symptom and sign. It describes a condition presenting as anaesthesia or paraesthesia over the chin, which is usually unilateral. The condition manifests spontaneously with no history of trauma, infection or obvious odontogenic cause. NCS is a clinically important finding as it may be the primary manifestation of Multiple Sclerosis (MS) or sign of systemic malignancy. We present a case of a 58 -year-old gentleman who was referred to a regional oral and maxillofacial unit with a three-month history of sudden onset unilateral numbness of the chin. No odontogenic cause could be found and subsequent CT/PET scan from the cerebellum to the upper thighs revealed evidence of widespread metastatic disease. The patient died five weeks after his initial presentation. We discuss the importance of this clinical symptom, the likely mechanism of disease and offer differential diagnoses. Additionally, we recommend that patients presenting to their general dental practitioner with a numb chin be urgently investigated for a potentially undiagnosed malignancy or MS and be referred to their local oral and maxillofacial unit as an urgent 'target' referral.

\section{INTRODUCTION}

Numb chin syndrome (NCS) is a condition where there is altered sensation along the distribution of the mandibular branch of the trigeminal nerve without any obvious odontongenic cause. ${ }^{1}$ NCS commonly presents as anaesthesia or paraesthesia over the chin and is usually unilateral. The condition manifests spontaneously with no history of trauma, infection or local dental disease. ${ }^{2}$ NCS is a clinically important finding as it may be a manifestation of MS or the first sign of systemic malignancy. ${ }^{3-7}$ It has been described as the first presenting symptom in small cell lung cancer, ${ }^{3}$ breast cancer, ${ }^{3}$ prostate cancer, ${ }^{4}$ lymphoma ${ }^{5}$ and malignant melanoma. ${ }^{6}$

It should be emphasised that any neuropathy of the mental nerve that does not have an obvious dental cause should be

${ }^{1}$ Clinical Fellow, Oral and Maxillofacial Surgery, ${ }^{2 *}$ Clinical Fellow, Oral and Maxillofacial Surgery, ${ }^{3}$ Consultant Oral and Maxillofacial Surgeon, Oral and Maxillofacial Surgery, Bart's and the Royal London NHS Trust, Whitechapel, London, E1 1BB

*Correspondence to: Dr Scott Rice

Email:scott.rice@mac.com

\section{Refereed Pape}

Accepted 19 February 2010

DOI: $10.1038 /$ sj.bdj.2010.292

${ }^{\bullet}$ British Dental Journal 2010; 208: 283-285

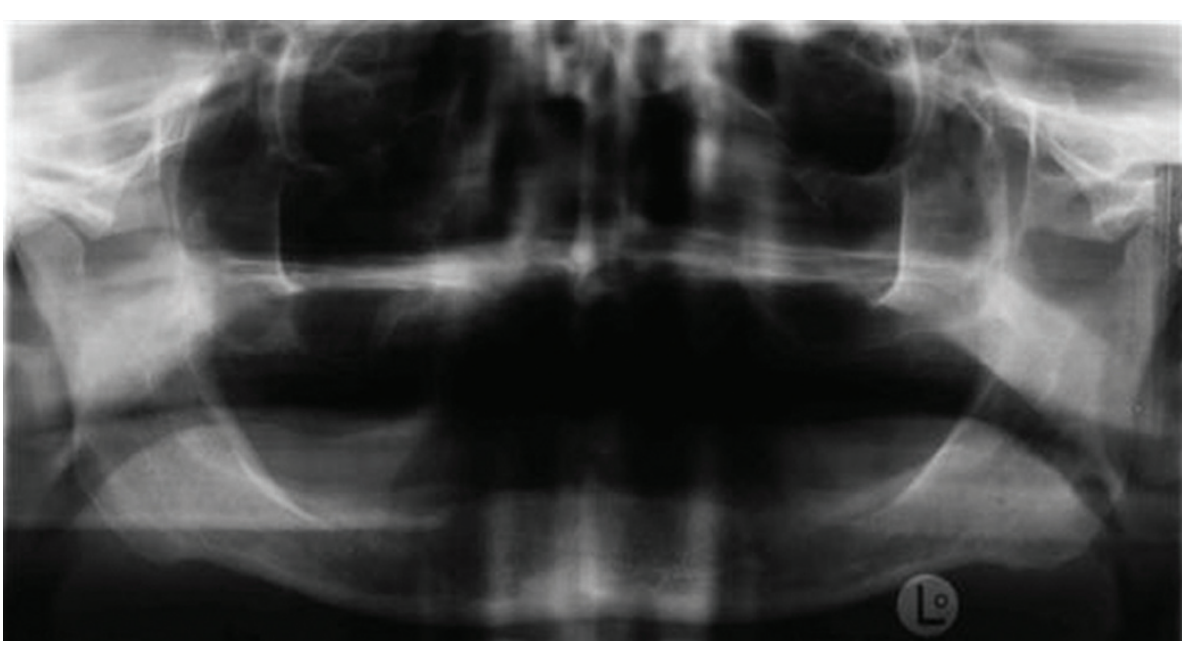

Fig. 1 Patient's dental panoramic radiograph on initial examination

considered highly suspicious of malignancy and investigated aggressively.

\section{CASE REPORT}

A 58-year-old man was referred by his general dental practitioner (GDP) with a threemonth history of unilateral anaesthesia of his left chin and lip and a ten-week history of dull, throbbing pain from his left jaw radiating to his ear. He described a sudden onset of paraesthesia over a small area of the left side of his chin. This had progressively increased to involve his entire chin, lip and the oral mucosa overlying the left mandible.
His medical history revealed that he suffered from ulcerative colitis, for which he was taking sulphalazine (500 $\mathrm{mg}$, six times per day), mercaptopurine (50 mg, twice daily) and pantoprazole (40 mg, once daily), and emphysema. He stopped smoking nine months earlier but previously smoked 40 cigarettes a day for 20 years (40 pack years). He did not drink alcohol.

On examination he was edentulous with no obvious mucosal pathology or cervical lymphadenopathy. Neurological examination revealed complete sensory disturbance along the distribution of the left inferior 
dental and mental nerve $\left(\mathrm{CrN} \mathrm{V}_{3}\right)$ with lingual nerve sparing. All other cranial nerves were intact, there were no cerebral or cerebellar abnormalities and the peripheral nervous examination was normal. A dental panoramic radiograph (Fig. 1) was unremarkable with no evidence of focal pathology. Haematological investigation revealed a macrocytic anaemia $(\mathrm{Hb} 8.2 \mathrm{~g} / \mathrm{dL})(\mathrm{MCV}$ 100.4fl) associated with neutrophilia (8.5 $\left.10^{9} / \mathrm{L}\right)$, lymphocytopenia $\left(0.510^{9} / \mathrm{L}\right)$ and raised platelets $\left(65710^{9} / \mathrm{L}\right)$. He had a raised alkaline phosphatase (146 iu/L) and raised ferritin levels (276 ug/L). A positron emission tomography study combined with computed tomography (PET/CT) from the cerebellum to the upper thighs demonstrated increased uptake throughout most bones. This was particularly evident in the left temporomandibular joint (TMJ), the left mandible (Fig. 2) and throughout the cervical, thoracic and lumbar spine. There was marked destruction in the iliac bone, multiple ribs and sternum. Additionally, there was focal uptake within several pulmonary nodules, particularly in the right upper lobe. This suggested widespread metastatic disease, although there was no obvious primary site.

Five days following his outpatient PET/ CT scan he was admitted to his local hospital with an acute exacerbation of ulcerative colitis and suspected pneumonia. Magnetic resonance (MR) imaging of his spine undertaken locally confirmed multiple vertebral metastases. Further CT scanning failed to demonstrate a primary site of malignancy but showed abnormalities in the lungs, liver, ascending colon and a large soft tissue density mass in the pelvis with destruction of the left iliac bone.

Unfortunately, the patient's condition deteriorated quickly and a planned tissue biopsy could not be done. He died five weeks after his initial presentation to the regional OMFS unit. The family decided not to have a post mortem examination and therefore the primary cause of his metastatic disease was never confirmed.

\section{DISCUSSION}

Numb chin syndrome (also known as mental nerve neuropathy) is a rare but important clinical symptom and sign. In this case there was no obvious local cause for the patient's numb chin. The patient was edentulous and radiographic examination of

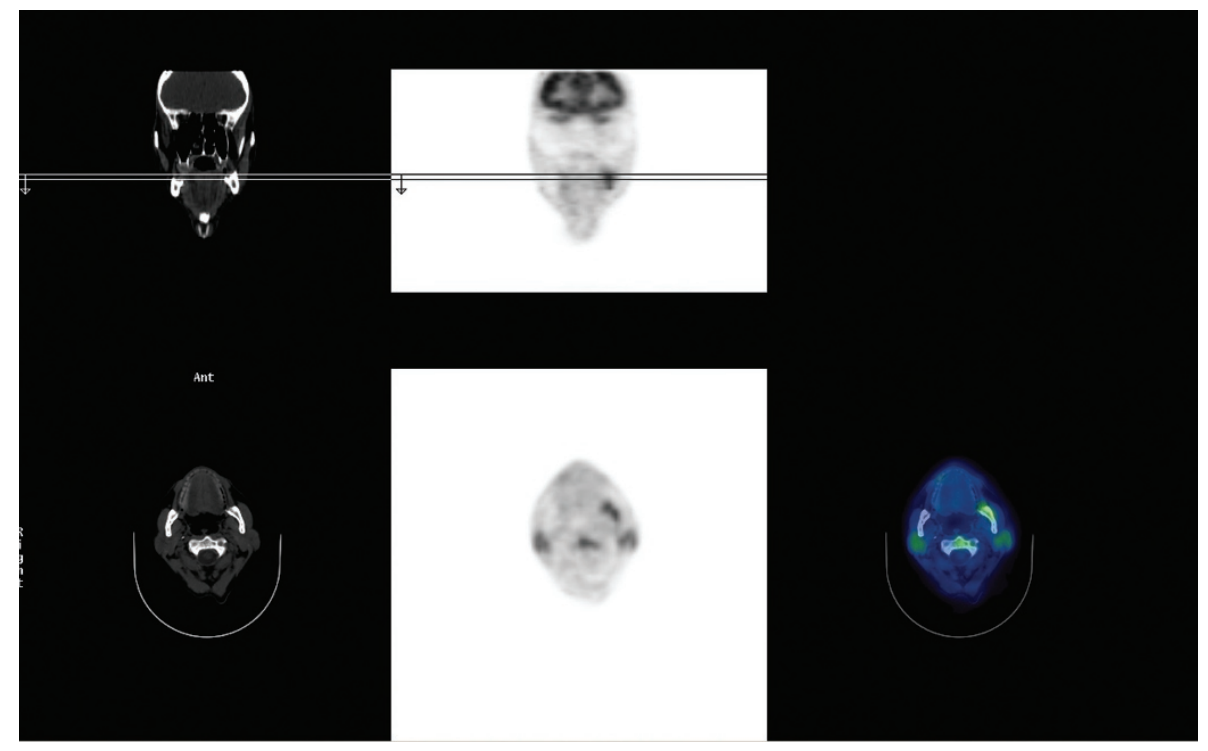

Fig. 2 PET/CT scan of the left mandible showing increased uptake in the left mandible

the mandible did not show any abnormalities. However, a PET/CT scan suggested the presence of undiagnosed widespread metastatic bone deposits, a finding supported by the raised serum alkaline phosphatase. PET involves intravenous injection of a glucose derivative (Fluorodeoxy glucose or FDG) radioactively labelled with short-lived positrons generated in Cyclotron machines. The FDG is taken up by metabolically active cells and is a marker for inflammation and infection as well as neoplasia. It is non-specific. However, neoplastic tissues take up FDG more avidly and therefore show up more densely on scans. The anatomical specificity of the PET scan is not as good as with CT or MRI so co-location PET/CT scans help in more accurate localisation of the abnormal cells. ${ }^{7}$

The mandibular branch of the trigeminal nerve runs through a bony canal in the mandible, entering at the mandibular foramen and exiting at the mental foramen.

NCS is almost always unilateral in presentation and is associated with altered sensation along the anatomical boundary of the nerve, most commonly the mental division. It not associated with any motor or taste disturbance. ${ }^{3}$ It may have local odontogenic causes such as infection, trauma (direct and indirect), dental local anaesthetic and odontogenic neoplasms. ${ }^{8}$ It may also be a consequence of radiotherapy to the mandible causing direct damage to the nerve or by ischaemia. ${ }^{1}$ It may be caused by compression of the nerve through the infratemporal fossa and skull base or compression of the

Table 1 Non-malignant causes of
a numb chin
Trauma at site of inferior dental nerve block
Orthognathic surgery
Mandibular trauma
Haematoma
Ondontogenic infection
Mandibular osteomyelitis
Root canal filling with root filling material pro-
truding beyond apex
Multiple sclerosis
Chronic apical periodontitis
Root cysts
Severe mandibular atrophy
Benign sensory trigeminal neuropathy
Radiotherapy
Chemical exposure
Viral infection
Post-vaccination vasculitis
Connective tissue vasculitis
Systemic amyloidosis
Sickle cell disease
Sarcoidosis
HIV
Diabetes mellitus
Lyme disease
Syphilis
Vertebrobasilar insufficency

nerve root through its intracranial course by direct causes or indirectly through a rise in intracranial pressure. Alternatively selective demyelination of the nerve in MS can cause NCS as an isolated neurological sign. Other non-malignant causes of a numb chin are summarised in Table 1. Of note, the use of metronidazole has also been implicated as a cause of unexplained neuropathy. ${ }^{9}$ The exact mechanism of toxicity is unclear, however, 
one hypothesis is that it is secondary to metronidazole binding to ribonucleic acid and inhibiting protein synthesis. ${ }^{10}$

The mechanism by which mental nerve neuropathy occurs as a consequence of metastatic disease is not well understood. There are several hypotheses to explain the sensory defect, the most widely accepted being nerve compression by mandibular bone metastasis., ${ }^{2,3}$ Other hypotheses include perineural invasion of the trigeminal nerve and its branches, ${ }^{11}$ spread to the central nervous system, ${ }^{3}$ intracranial involvement due to metastasis to the base of skull ${ }^{12}$ and leptomeningeal seeding. ${ }^{13}$

Numb chin syndrome in the context of metastatic disease is a serious diagnosis with a poor prognosis. Only 15\% of patients who present with NCS and who are found to have metastatic disease survive more than nine months. ${ }^{2,3,13}$ This is because NCS is usually a very advanced symptom of metastatic disease and, in the absence of a benign cause, the sign is always indicative of disease progression. Review of the literature reveals that NCS precedes diagnosis of malignancy in up to $47 \%$ of cases. ${ }^{3}$ Some reports have suggested that patients fail to seek treatment as it is a minor symptom ${ }^{2}$ and, additionally, because a numb chin is not in itself a very serious condition with significant morbidity, it may be overlooked or not urgently investigated by practitioners.

\section{CONCLUSION}

Patients who present with a sudden alteration in sensation along the distribution of the mandibular branch of the trigeminal nerve, the so-called 'numb chin syndrome', in the absence of an obvious cause, should be urgently investigated for undiagnosed malignancy with a low threshold for immediate PET/CT imaging and MR imaging to exclude MS. This report demonstrates the need to investigate this seemingly trivial symptom aggressively because it can herald associated metastatic disease with a very poor prognosis. Although in this case we were not able to determine the primary site of the cancer, it highlights, on top of already documented evidence, the numb chin as an important clinical sign. We therefore recommend that general dental practitioners refer such patients as urgent 'cancer-target' referrals to their local oral and maxillofacial unit for investigation without delay.

1. Lossos A, Siegal T. Numb chin syndrome in cancer patients: etiology, response to treatment and prognostic significance. Neurology 1992; 42: 1181-1184.

2. Sanchis J M, Began J V, Murillo J et al. Mental neuropathy as a manifestation associated with malignant processes: its significance in relation to patient survival. J Oral Maxillofac Surg 2008; 66: 995-998.

3. Laurencet F M, Anchisi S, Tullen E, Dietrich P Y. Mental neuropathy: report of five cases and review of the literature. Crit Rev Oncol Hematol 2000; 34: 71-79.

4. Gaver A, Polliack G, Pilo R, Hertz M, Kitai E. Orofacial pain and numb chin syndrome as the presenting symptoms of a metastatic prostate cancer. J Postgrad Med 2002; 48: 283-284.

5. Baskaran R K, Krishnamoorthy, Smith M. Numb chin syndrome - a reflection of systemic malignancy. World J Surg Oncol 2006; 4: 52-54.

6. Pruckmayer M, Glaser C, Marosi C, Leitha T. Mandibular pain as the leading clinical symptom for metastatic disease: nine cases and review of the literature. Ann Oncol 1998; 9: 559-564.

7. Hardee P S G F, Hutchison I L. Solitary nodal metastases presenting as branchial cysts: a diagnostic pitfall. Ann R Coll Surg Engl 1999; 81: 296-298.

8. Mallefert J F, Gazet-Maillefert M P, Tavernier C, Farge P. Numb chin syndrome. Joint Bone Spine 2000; 67: 86-93.

9. Hobson-Webb L D, Roach E S, Donofrio P D. Metronidazole: newly recognized cause of autonomic neuropathy. J Child Neurol 2006; 21: 429-431.

10. Bradley W G, Karlsson I J, Rassol C G. Metronidazole neuropathy. Br Med J 1977; 3: 610-611.

11. Eppley B L, Snyders R V. Mental neuropathy as a sign of distant malignancy: report of cases. J Oral Maxillofac Surg 1992; 50: 1117-1119.

12. Kuntzer T, Bogousslavsky J, Rilliet B, Uldry P A et al. Herald facial numbness. Eur Neurol 1992; 32: 297-301.

13. Penarrocha Diago M, Bagan Sebastian J V, Alfaro Giner A, Escrig Orenga V. Mental nerve neuropathy in systemic cancer. Oral Surg Oral Med Oral Pathol 1990; 69: 48-51. 